\title{
Preoperative staging in childhood craniopharyngioma: standardization as a first step towards improved outcome
}

\author{
Hermann L. Müller ${ }^{1}$
}

Received: 23 September 2015/ Accepted: 4 November 2015/Published online: 18 November 2015

(C) Springer Science+Business Media New York 2015

\section{Introduction}

Childhood-onset craniopharyngiomas (CP) are classified by WHO as brain tumors of low-grade histological malignancy. During the last decades, the prognosis of pediatric patients with low-grade brain tumors of other histology has been continuously und substantially improved by implementation of standardized treatment protocols used in national and international trials. A mainstay of this success-illustrated by improved survival and outcome- has been the standardized staging at primary diagnosis. However, in CP such national and international efforts on standardization of preoperative staging and treatment strategies are still missing.

CPs are rare embryonal malformations. Hypothalamic involvement and/or treatment-related lesions result in impaired physical and social functionality and severe neuroendocrine sequelae [1]. Quality of life in CP with hypothalamic involvement is impaired by severe obesity, physical fatigue, reduced motivation, dyspnea, diarrhea, and non-optimal psychosocial development [2]. Patients with CP involving hypothalamic structures showed reduced 10 -years overall survival, whereas overall and progressionfree survival rates are not related to the degree of surgical resection [2]. However, these results are based on a multicenter study that spans nearly five decades during which dramatic changes in surgical and radiation therapy

Hermann L. Müller

mueller.hermann@klinikum-oldenburg.de;

http://www.kraniopharyngeom.net

1 Department of Pediatrics, Klinikum Oldenburg, Medical Campus University Oldenburg, Rahel-Straus-Strasse 10, 26133 Oldenburg, Germany occurred. In fact, there was no uniform treatment paradigm and no assessment of relative surgical (or radiation oncology) experience, which has been reported as a critical factor for long-term outcome [3-5]. Furthermore, contemporary series show relatively equivalent 5 - and 10-year progression-free survival rates associated with radical surgery or partial resection combined with irradiation. Information on the impact of salvage therapies on survival and quality of life in the 20-30\% of patients with recurrent disease is limited.

Several grading systems for assessment of pre and/or postoperative location and hypothalamic involvement of $\mathrm{CP}$ and algorithms for specific treatment strategies based on these grading systems have been published [4-11]. Mortini et al. [12] are publishing an analysis of their single center cohort of exclusively adult-onset CPs comparing three of the above-mentioned grading systems [4-6, 9] in terms of prediction value for outcome and the development of hypothalamic syndrome.

\section{Grading systems for hypothalamic involvement/ lesions in $\mathbf{C P}$}

Novel risk-adapted treatment strategies are reportedly focusing on the following main goals: (a) relief of raised intracranial pressure, (b) reversal of visual compression symptoms, (c) restoration or substitution of pituitary hormone deficits plus all other supplement-supportive measures, and (d) prevention of tumor regrowth/progression, while minimizing acute and long-term morbidity and mortality.

De Vile et al. [10] published the first report on the association between attempts at radical resection in case of hypothalamic involvement and long-term morbidity. Puget 
et al. [6] published an algorithm for neurosurgical treatment of CP patients, which recommends a hypothalamussparing surgical strategy based on the grading of hypothalamic tumor involvement in preoperative magnetic resonance imaging (MRI). The authors reported that patients treated according to this algorithm using a hypothalamus-sparing surgical approach had similar relapse rates and a lower prevalence of severe obesity than patients treated by gross-total resection (28 vs $54 \%$, respectively). This was the first report in the literature proving the efficacy and tolerability of a hypothalamussparing strategy by comparing cohorts treated at a single institution by the same experienced surgical team, and thus eliminating the bias of surgical experience on outcome analysis. However, it is important to note that although the "hypothalamus-sparing surgery" increased the percentage of "normal" BMI from 17 to $38 \%$, the likelihood of significant weight gain remained $62 \%$ with nearly half of all patients developing morbid obesity. Garré et al. [8] used this grading system of hypothalamic involvement and published a modified algorithm for risk-adapted treatment strategies in CP. The authors emphasized the treatment by experienced neurosurgical teams and suggested proton beam therapy, especially for young patients $(<5$ year of age) after limited hypothalamus-sparing surgery. Müller et al. $[4,5]$ suggested a treatment strategy based on preand post-surgical grading of hypothalamic tumor involvement/damage in MRI. The assessment of the tumor extension towards the mammillary bodies is considered essential for the grading into anterior or posterior hypothalamic involvement/lesion. According to their report, patients with post-surgical lesions of the posterior hypothalamus presented with a higher BMI and lower selfassessed quality of life during prospective follow-up. Mallucci et al. [7] published a treatment algorithm, suggesting a two-staged surgical approach with initial relief of cystic pressure and thereby down-staging the risk grade in appropriate cases. Van Gompel et al. [9] developed an MRI-based grading system to predict risk of postoperative obesity in adults treated for CP. Hypothalamic T2 signal change and irregular contrast enhancement correlated and predicted higher-grade hypothalamic involvement. Progressive hypothalamic involvement correlated with larger postoperative weight gains. Steno et al. [13] reported a higher hypothalamic complication rate, in case of tumors with intraventricular extension as compared with purely extraventricular tumors. To preserve the hypothalamic function, the authors recommend to distinguish between suprasellar extraventricular and suprasellar intra- and extraventricular craniopharyngioma. In the first group, the hypothalamus can be simply compressed and not infiltrated. Elliott et al. [11] used a newly designed craniopharyngioma clinical status scale (CCSS) to show that preoperative CCSS scores predicted postoperative outcome better than clinical characteristics like patient age, sex, tumor size, and the location or presence of hydrocephalus.

All of the above-mentioned treatment strategies recommend that (a) for cases of hypothalamic tumor involvement, limited surgical approaches and postoperative radiotherapy are advisable, and (b) treatment of CP should be confined to experienced multidisciplinary teams.

\section{Comparison of grading systems in $\mathrm{CP}$}

A major step towards potential standardization of preoperative staging in $\mathrm{CP}$ is the comparison of published grading systems for assessment of hypothalamic involvement/damage in regard to prediction value for severe hypothalamic obesity as the main sequelae impairing quality of survival in this disease. Mortini et al. [12] are publishing one of the first studies, which identifies radiological variables linked to hypothalamic involvement on the preoperative MRI, and correlate them using statistical criteria, with clinical features, long-term outcome, and prognosis. Furthermore, the authors analyze the sensitivity of three published grading systems [4-6,9] for prediction of hypothalamic obesity in their single center cohort. Hypothalamic hyperintensity in T2-weighted/FLAIR images, mammillary body involvement, unidentifiable pituitary stalk, dislocated chiasm, either infundibular recess or unrecognizable supra-optic recess, and retrochiasmatic tumor extension have proved to be useful to define the hypothalamus invasion. Variables identified as factors with high and comparable prediction value for postoperative hypothalamic syndrome were the degree of hypothalamic involvement according to the classification described by Sainte-Rose and Puget [6] $(p<0.002$; grade 0 vs 2 $p<0.001)$, van Gompel et al. [9] ( $p<0.002$; grade 0 vs 1 , $p<0.027$; and grade 0 vs $2, p<0.002$ ), and Muller et al. $[4,5](p<0.006$; grade 0 vs $1, p<0.05$; and grade 0 vs 2 , $p<0.004)$. These results impressively support the hypothesis that disease or treatment-related hypothalamic alterations have major negative impact on prognosis and quality of survival in $\mathrm{CP}$.

\section{Conclusions}

Mortini et al. [12] confirm that current grading systems for assessment of hypothalamic involvement/damage in CP are clinically useful tools with comparable and high predictive value in estimation of risk for hypothalamic obesity. Hopefully their important observation supports efforts in establishing standards for staging in $\mathrm{CP}$, which should be implemented by national and international societies. Other 
aspects of severe hypothalamic dysfunction including hyperphagia and behavioral disturbances should also be taken into consideration for future studies. Based on the current literature, an attempt at radical resection at the time of primary diagnosis with respect to preservation of hypothalamic and visual integrity especially in case of initial hypothalamic involvement seems to be the treatment of choice.

Acknowledgments This manuscript was composed in the absence of any commercial or financial relationships that could be construed as a potential conflict of interest. The author is supported by the German Childhood Cancer Foundation, Bonn, Germany.

\section{References}

1. H.L. Muller, Craniopharyngioma. Endocr. Rev. 35(3), 513-543 (2014). doi:10.1210/er.2013-1115

2. A.S. Sterkenburg, A. Hoffmann, U. Gebhardt, M. WarmuthMetz, A.M. Daubenbuchel, H.L. Muller, Survival, hypothalamic obesity, and neuropsychological/psychosocial status after childhood-onset craniopharyngioma: newly reported long-term outcomes. Neuro-oncology 17(7), 1029-1038 (2015). doi:10.1093/ neuonc/nov044

3. R.A. Sanford, Craniopharyngioma: results of survey of the American Society of Pediatric Neurosurgery. Pediatr. Neurosurg. 21(Suppl 1), 39-43 (1994)

4. H.L. Muller, U. Gebhardt, A. Faldum, M. Warmuth-Metz, T. Pietsch, F. Pohl, G. Calaminus, N. Sorensen, Xanthogranuloma, Rathke's cyst, and childhood craniopharyngioma: results of prospective multinational studies of children and adolescents with rare sellar malformations. J. Clin. Endocrinol. Metab. 97(11), 3935-3943 (2012). doi:10.1210/jc.2012-2069

5. H.L. Muller, U. Gebhardt, C. Teske, A. Faldum, I. Zwiener, M. Warmuth-Metz, T. Pietsch, F. Pohl, N. Sorensen, G. Calaminus, Post-operative hypothalamic lesions and obesity in childhood craniopharyngioma: results of the multinational prospective trial
KRANIOPHARYNGEOM 2000 after 3-year follow-up. Eur. J. Endocrinol./Eur. Fed. Endocr. Soc. 165(1), 17-24 (2011). doi:10.1530/EJE-11-0158

6. E. Elowe-Gruau, J. Beltrand, R. Brauner, G. Pinto, D. SamaraBoustani, C. Thalassinos, K. Busiah, K. Laborde, N. Boddaert, M. Zerah, C. Alapetite, J. Grill, P. Touraine, C. Sainte-Rose, M. Polak, S. Puget, Childhood craniopharyngioma: hypothalamussparing surgery decreases the risk of obesity. J. Clin. Endocrinol. Metab. 98(6), 2376-2382 (2013). doi:10.1210/jc.2012-3928

7. C. Mallucci, B. Pizer, J. Blair, M. Didi, A. Doss, S. Upadrasta, W. Newman, S. Avula, B. Pettorini, Management of craniopharyngioma: the Liverpool experience following the introduction of the CCLG guidelines. Introducing a new risk assessment grading system. Child's Nerv. Syst. 28(8), 1181-1192 (2012). doi:10. 1007/s00381-012-1787-8

8. M.L. Garre, A. Cama, Craniopharyngioma: modern concepts in pathogenesis and treatment. Curr. Opin. Pediatr. 19(4), 471-479 (2007). doi:10.1097/MOP.0b013e3282495a22

9. J.J. Van Gompel, T.B. Nippoldt, D.M. Higgins, F.B. Meyer, Magnetic resonance imaging-graded hypothalamic compression in surgically treated adult craniopharyngiomas determining postoperative obesity. Neurosurg. Focus 28(4), E3 (2010). doi:10. 3171/2010.1.FOCUS09303

10. C.J. De Vile, D.B. Grant, B.E. Kendall, B.G. Neville, R. Stanhope, K.E. Watkins, R.D. Hayward, Management of childhood craniopharyngioma: can the morbidity of radical surgery be predicted? J. Neurosurg. 85(1), 73-81 (1996). doi:10.3171/jns. 1996.85.1.0073

11. R.E. Elliott, S.A. Sands, R.G. Strom, J.H. Wisoff, Craniopharyngioma Clinical Status Scale: a standardized metric of preoperative function and posttreatment outcome. Neurosurg. Focus 28(4), E2 (2010). doi:10.3171/2010.2.FOCUS09304

12. P. Mortini, F. Gagliardi, M. Bailo, A. Spina, A. Parlangeli, A. Falini, M. Losa, Magnetic resonance imaging as predictor of functional outcome in craniopharyngiomas. Endocrine (2015). doi:10.1007/s12020-015-0683-x

13. J. Steno, I. Bizik, A. Steno, V. Matejcik, Craniopharyngiomas in children: how radical should the surgeon be? Child's Nerv. Syst 27(1), 41-54 (2011). doi:10.1007/s00381-010-1330-8 